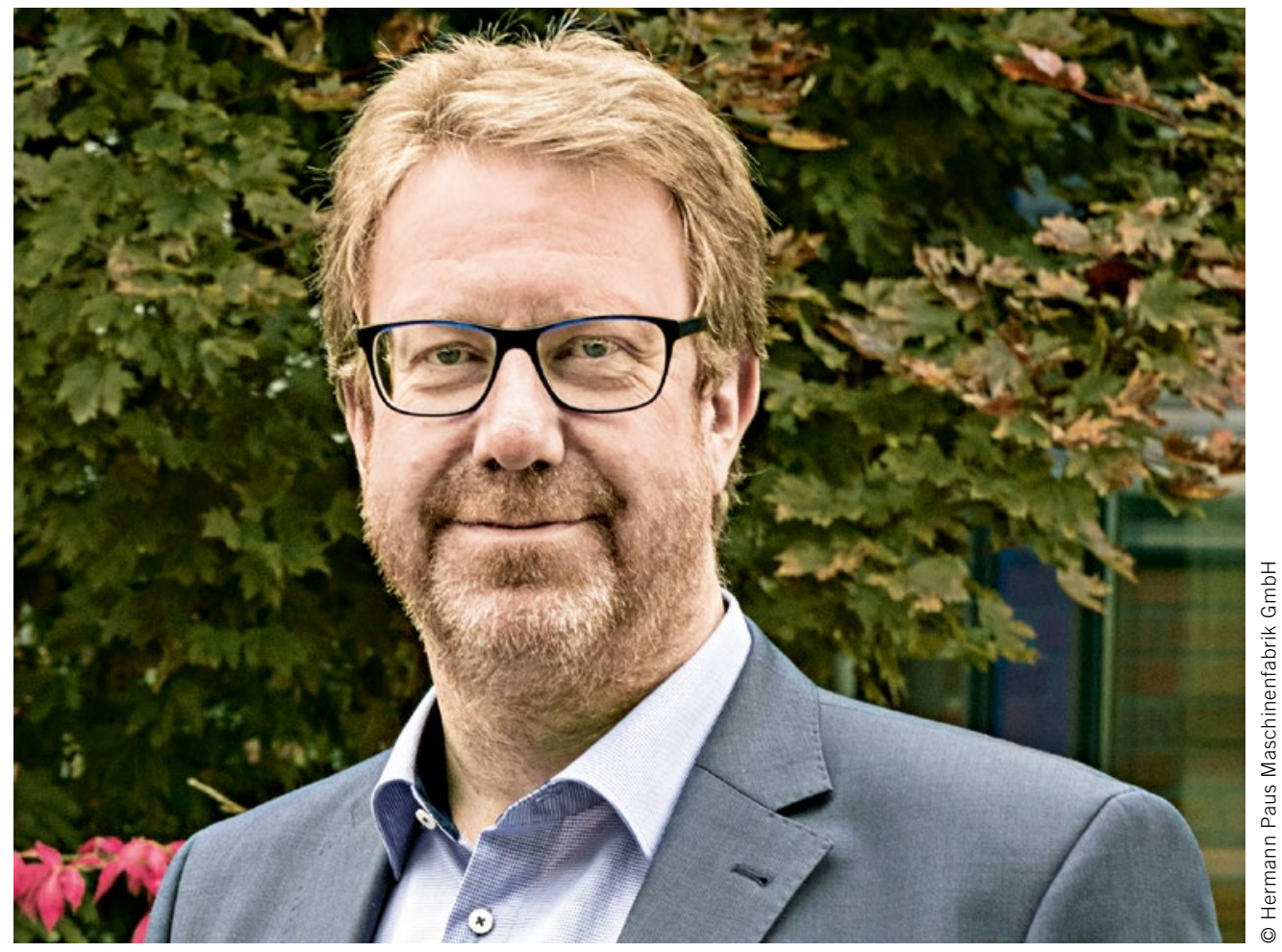

Franz-Josef Paus

Chairman of the VDMA

Construction Equipment and

Plant Engineering Association and Executive Manager of

Hermann Paus Maschinenfabrik

$\mathrm{GmbH}$ in Emsbüren (Germany)

\title{
Confident and Crisis-tested
}

Despite declining incoming orders, the construction equipment sector has not been as badly affected by the Covid-19 crisis as other sectors. Although it is difficult to make predictions in the current situation, we at VDMA have managed to draw quite a clear picture of the sentiment across sectors, including construction equipment, thanks to regular surveys conducted since February. Since June, disruptions in the supply chain have had no serious impact. Overall, nobody believes that it will take longer than four years before the sector has completely recovered. It is still benefiting from the boom years of 2018 and 2019 and was able to support itself in the first six months of the year with existing orders and sales. Many construction sites remained up and running, with projects being rescheduled rather than being canceled; a factor which has also contributed to the overall stability of the sector. Manufacturers of construction equipment reacted flexibly to the new circumstances, in part adapting their production to multi-shift operation, thereby relieving the pressure on their employees and adhering to the hygiene measures. Unfortunately, some cases of Covid-19 were diagnosed among the workforce and closures and short-time work could not be entirely avoided in Germany. Hope has now been placed in the economic programs.

The thoughts are directed positively into the future, as climate goals, progressive building, autonomous construction sites and digitalization are all factors which offer market poten- tial that should be developed and used. Construction equipment is becoming ever cleaner, quieter and more intelligent. The spirit with which we go about our research is as high as ever, and we know that we can only counteract international political heavyweights and trade barriers with technological expertise and, above all, by taking a leading role. And we know that we cannot do this alone. Within VDMA, we are working at full speed on future-proof concepts and projects on both a national and European level - such as on a standardized machine language for mobile machines in the MIC 4.0 working group. The German Federal Ministry of Education and Research has sponsored the Bauen 4.0 project within the Industrie 4.0 program - Collaborations in Dynamic Value Creation Networks. The technical universities in Dresden and Munich, as well as a consortium of 20 companies and five research associations, are collaborating to research machine and communication technologies. Until July 2022, they are developing a demonstration and development platform focusing on "automated, connected mobile machines," "5G machine and construction site connectivity" and "processes and solutions for the digital construction site.” The three areas will be connected and the goal is for them to complete a construction task together. The project aims to support medium-sized companies in implementing digitalized processes at construction sites in particular. 